\title{
1 The human oral phageome is highly diverse and rich in jumbo phages
}

3 Victoria R. Carr ${ }^{1,2 *}$, Andrey Shkoporov ${ }^{3 *}$, David Gomez-Cabrero ${ }^{4}$, Peter Mullany ${ }^{5}$, Colin

4 Hill $^{3}$, David L. Moyes ${ }^{1}$

$5 \quad{ }^{1}$ Centre for Host-Microbiome Interactions, Faculty of Dentistry, Oral and Craniofacial

6 Sciences, King's College London, UK

$7 \quad{ }^{2}$ The Alan Turing Institute, British Library, London, UK

$8 \quad{ }^{3}$ APC Microbiome Ireland \& School of Microbiology, University College Cork, Ireland

$9{ }^{4}$ Translational Bioinformatics Unit, NavarraBiomed, Departamento de Salud-Universidad

10 Pública de Navarra, Pamplona, 31008, Navarra, Spain

$11{ }^{5}$ Microbial Diseases, Eastman Dental Institute, University College London, UK

$12 *$ These authors contributed equally to the work

13 Correspondence and requests for materials: victoria.carr@kcl.ac.uk, david.moyes@kcl.ac.uk

\section{Keywords}

16 Phage; human microbiome; phageome; bacteriophage; jumbo phage; oral cavity; gut;

17 gastrointestinal tract

\section{Abstract}

20 Until recently, the contribution of bacteriophages to the composition and function of the

21 human microbiome has been largely overlooked. Recent developments in discovering novel

22 bacteriophages from human metagenomes have been mostly focused on the gut. Here we

23 profile and compare the phageome of 633 human oral sites and 221 paired gut phageomes

24 acquired from countries worldwide. Phageome profiles are specific to oral sites and the gut

25 across individuals, with the largest separation between the oral cavity and the gut. The 
26 differences in phage genotypes is bolstered by distinct phage host compositions between

27 these sites. The greatest diversity of phages is found on the tongue and in dental plaque.

28 Notably, 37 unique circular jumbo phage genomes were identified in oral sites, particularly

29 on the dorsum of the tongue, whilst none were found in the gut. Oral sites provide conducive

30 environments, such as robust biofilms, that can harbour genetically diverse phages.

\section{Introduction}

33 Bacteriophages are the most abundant viral components of the human microbiome. They are

34 viruses that infect and replicate their genome within bacterial or archaeal cells, and are likely

35 to have significant effects on microbial composition and function ${ }^{1,2}$. Like eukaryotic viruses,

36 they can have single or double stranded DNA or RNA genomes. They also have two

37 principal life cycles: virulent, which destroy bacterial cells immediately after replication; and

38 temperate, which have the option of integrating their genome into the host genome

39 (lysogeny) and are involved in the horizontal gene transfer of many genetic elements,

40 including virulence factors.

42 Many studies that attempt to profile bacteriophages in human microbiomes use computational

43 analysis of faecal metagenomic data, often following enrichment of virus-like particles ${ }^{3,4}$.

44 This has led to the discovery of hundreds of thousands of novel and uncultivated virus

45 genomes ${ }^{5}$. These include crAssphages, a highly abundant bacteriophage clade currently

46 thought to play a special role in human and primate gut microbiomes ${ }^{6-8}$; the discovery of

47 atypically large bacteriophages greater than $200 \mathrm{~kb}$ in length, known as jumbo phages ${ }^{9}$; and

48 more recently megaphages with genomes larger than $500 \mathrm{~kb}^{10}$. Only a handful of studies have

49 profiled bacteriophages using metagenomic data from the oral cavity ${ }^{11,12}$. The heterogeneity

50 of bacteriophage genomes, and lack of correlation between phage phylogeny and that of their 
51 hosts, makes classification and host assignment challenging tasks, leaving a relatively

52 unexplored melting pot of "viral dark matter"3,13.

54 Here, we profile bacteriophage DNA from different locations in the human gastrointestinal

55 tract, specifically comparing gut (represented by faecal samples) with paired saliva and dental

56 plaque from China, and the dorsum of tongue, dental plaque and buccal mucosa from

57 longitudinal samples from the USA. We identify novel bacteriophages, including jumbo

58 phages, from assembled metagenomic contigs using de novo bioinformatic pipelines,

59 including viral motif recognition ${ }^{14}$ and protein-coding gene-sharing networks ${ }^{15}$ to identify

60 and classify linear and circular viral contigs. Bacteriophage hosts are also predicted using

61 CRISPR spacer matches with reference bacterial genomes ${ }^{16}$.

63 We report that bacteriophages and their host profiles are specific to gastrointestinal tract

64 (GIT) sites, with more differences found between the oral cavity and the gut than between

65 different oral sites. The dorsum of the tongue contains a greater diversity of bacteriophages

66 than paired stool samples, saliva and buccal mucosa. Consistent with greater genotypic

67 diversity, 37 unique circular jumbo phage genomes were found in oral cavities from China

68 and the USA, mainly on the dorsum of the tongue from the USA, while none were identified

69 in paired stool samples. In addition, bacteriophages can persist in some individuals for

70 months at a time, including phage families that are common to both oral cavity and gut, or are

71 specific to a GIT site, such as the crAss-like family of the gut, Inoviridae of the oral cavity,

72 and Microviridae of the gut, dorsum of the tongue and saliva.

\section{Results}

\section{Phage composition and diversity differs between GIT sites}


76 A catalogue of 139,929 phage contigs were identified from 854 oral and gut metagenomes.

77 Phage contigs were grouped into clusters using vConTACT2 ${ }^{15}$, a software based on gene-

78 sharing profiles that has been applied to stratify uncultured viral populations in a variety of

79 environments, including human gut metagenomes ${ }^{3,16} \cdot 75,680$ phage contigs were clustered

80 into 14,078 phage clusters, while the remaining 64,249 remain as singletons. It is more

81 common to find phage contigs that are unique to one sample than phage clusters found,

82 meaning phage contig profiles at strain level are more variable than phage cluster profiles

83 (Supplementary Fig. 1). $\beta$-diversity was evaluated with Bray-Curtis dissimilarity metric from

84 phage cluster abundance profiles to investigate differences between metagenomes. Samples

85 were clustered using unsupervised Silhouette analysis on k-medoids of non-metric

86 multidimensional scaling (NMDS) dimensions. Four groups were generated (Fig. 1a). Most

87 phage clusters are exclusively found in either Group 2, 3 or 4 or are shared between Group 2

88 and 3 (Fig. 1b). The composition of these groups is dominated by samples from a particular

89 GIT site (Fig. 1c). Groups 1 to 3 contain all oral sites: Group 1 consists of mostly buccal

90 mucosa, Group 2 contains mostly dorsum of tongue and saliva, and Group 3 has mostly

91 dental samples. In contrast, Group 4 contains exclusively stool samples. These results reveal

92 that many phage clusters are solely found in either dorsum of tongue (Group 2), dental

93 (Group 3) or stool samples (Group 4), but most phage clusters that are shared occur in both

94 dorsum of tongue and dental sites. Hierarchical clustering of the relative abundance of phage

95 clusters also shows GIT sites can be differentiated by phage cluster profiles, especially

96 between dental, dorsum of tongue and stool samples (Supplementary Fig. 2). GIT sites have

97 distinct phage cluster profiles, and phage clusters that are shared are mostly found in oral

98 sites. Incomplete metadata on sex and age meant that individual variation could not be

99 considered (Supplementary Fig. 3). 
101 Additionally, the $\alpha$-diversity of the phage cluster composition was compared between paired

102 GIT sites from China and the USA. The Phage Cluster Richness, i.e. the number of unique

103 phage clusters, was evaluated for every paired sample. Dental plaque from the USA ( $\mathrm{p}=$

$\left.1042.84 \times 10^{-4}\right)$ and from China $(\mathrm{p}=0.0476)$, and dorsum of the tongue samples from the USA ( $\mathrm{p}$

$105=1.89 \times 10^{-7}$ ) have a significantly greater Phage Cluster Richness than stool samples (Two-

106 sided Wilcoxon Rank Sum Test; Fig. 1d). USA dorsum of the tongue samples also have a

107 significantly higher richness than dental plaque $(\mathrm{p}=0.0469)$. Buccal mucosa from the USA

108 and saliva from China have the lowest richness compared to all other paired GIT sites (saliva

109 vs. dental plaque from China: $\mathrm{p}=2.94 \times 10^{-6}$, saliva vs. stool from China: $\mathrm{p}=1.93 \times 10^{-4}$,

110 buccal mucosa vs. dental plaque from the USA: $p=3.91 \times 10^{-7}$, buccal mucosa vs. dorsum of

111 the tongue from the USA: $\mathrm{p}=1.26 \times 10^{-8}$, and buccal mucosa vs. stool from the USA: $\mathrm{p}=$

$1120.00175)$.

114 Most oral sites and stool metagenomes contain a high abundance of Siphoviridae, Myoviridae

115 and Podoviridae phage families but there are some distinctive differences in the less

116 abundant phage families between GIT sites (Supplementary Fig. 4). Bacterial hosts for

117 phages were predicted using Demovir (https://github.com/feargalr/Demovir). Although host

118 bacteria could only be predicted for $11.8 \%(16,513 / 139,929)$ of phage contigs, these highly

119 abundant phage families infect a range of bacterial genera (Supplementary Fig. 5). crAss-like

120 phage clusters are only found in stool samples and in Prevotella spp. as host, apart from two

121 saliva and two dorsum of the tongue samples (Supplementary Fig. 6). In contrast to the

122 crAss-like family, Inoviridae are found almost exclusively in oral sites and predicted to infect

123 Neisseria species. Microviridae are present in dorsum of the tongue, saliva and stool samples

124 but are far less prevalent in buccal mucosa and dental plaque. Prevotella in oral sites and

125 Faecalibacterium are the only genera predicted to contain Microviridae. Bicaudaviridae are 
only found in ten dorsum of the tongue samples and are represented by only one phage

128 Podoviridae and 64 crAss-like phage clusters (Supplementary Fig. 6). Across highly

129 abundant families (i.e. Siphoviridae, Myoviridae and Podoviridae), GIT sites are clustered by

130 the incidence of phage clusters suggesting that phage clusters of the same family are also

131 specific to GIT site. In terms of lower abundance families found across multiple GIT sites,

132 there is a pronounced separation of oral sites and stool in Microviridae phage clusters, with

133 some separation of oral sites by Inoviridae phage clusters. Notably, the Microviridae family

134 is dominated by a few phage clusters in oral sites in contrast to stool that contains many

135 different phage clusters.

\section{Phage hosts match varied microbial composition across GIT sites}

138 Bacteriophages have previously been shown to modulate the microbiota composition (in

139 particular the bacterial and archaeal composition) in the mouse gut via phage infection and

140 lysis of specific host bacteria ${ }^{2}$. To investigate whether microbial composition is associated

141 with distinctions in phageome profiles between GIT sites, bacterial and archaeal taxonomies

142 were profiled using MetaPhlAn $2^{17}$. Clustering of taxonomic composition is associated with

143 GIT site, with greater separation between oral sites and gut (Fig. 2a). Procrustes analysis was

144 applied to match corresponding points between phageome and taxonomic profiles.

145 Procrustean randomisation test (PROTEST) was then used to determine whether two profiles

146 showed significant association. Microbial composition and phageome profiles correlate and

147 co-locate by GIT site, especially between stool and dental samples (Fig. 2b) (0.70 to a

148 significance of $\mathrm{p}=0.001$ in PROTEST). Predicted phage hosts group by GIT site with very

149 little overlap between gut and oral sites (Fig 2c, Supplementary Fig. 7). Actinomyces,

150 Atopobium, Campylobacter, Fusobacterium, Neisseria and Rothia genera, that are mostly

151 found in the oral cavity (Supplementary Fig. 8), are predicted hosts of oral site phage (Fig 
152 2c). Likewise, Bacteroides, Bifidobacterium, Clostridium, Roseburia, Ruminococcus and

153 Parabacteroides genera found more exclusively in stool samples are also potential phage

154 hosts in the gut. However, Eubacterium, a bacterium mostly found in stool, and

155 Haemophilus, Prevotella, Streptococcus and Veillonella, bacteria mainly residing in the oral

156 sites, are prevalent across all oral sites and the gut. Upon closer inspection of the abundance

157 of these genera at a species level, there are some species that are more prevalent in either the

158 oral cavity or the gut. For instance, E. brachy and E. saphenum are species of Eubacterium

159 that are found almost exclusively in the oral cavity (Supplementary Fig. 9). Likewise, $P$.

160 copri mostly represents Prevotella, and is found almost exclusively in the gut. Generally,

161 phage host predictions match microbial composition at a genus level.

\section{Stability of phage clusters across longitudinal metagenomes}

164 To clarify whether phages that are associated with a GIT site are also stable over time, phage

165 clusters were profiled in longitudinal samples from the USA taken over a two-year period

166 with a minimum of two and maximum of six sampling timepoints. The proportions of total

167 phage clusters (Fig. 3a) and total reads mapped to these phage clusters (Fig. 3b) drops over

168 time across all GIT sites, but this is unclear from timepoints 4-6 in buccal mucosa, 4 in

169 dorsum of tongue and 5-6 in stool due to a small number of samples.

171 To compare the association between stable and unstable phage clusters and GIT site, the

172 longitudinal phageome was separated empirically into persistent and transient phage cluster

173 profiles. Persistent phage clusters are defined as being present in three or more timepoints in

174 a given GIT site, whereas transient phage clusters are found in less than three timepoints.

175 There are 194 (buccal mucosa), 1377 (dental plaque), 1423 (dorsum of the tongue) and 1899

176 (stool) persistent phage clusters, and 1323 (buccal mucosa), 2795 (dental plaque), 3246 
177 (dorsum of the tongue) and 2773 (stool) transient phage clusters. NMDS of persistent and

178 transient phage cluster profiles in an ordination of two-dimensions appears to show that

179 longitudinal samples are more clustered by individual in persistent (Fig. 3c) compared to

180 transient profiles (Fig. 3d). PERMANOVA (permutational multivariate analysis of variance)

181 was applied to persistent and transient phage cluster profiles for each GIT site to find which

182 category has the highest individuality. A greater percentage of variance of persistent than

183 transient phage clusters can be explained by individual variability across GIT sites. $78.1 \%$

184 (buccal mucosa), $72.3 \%$ (dental plaque), $78.4 \%$ (dorsum of the tongue) and $85.5 \%$ (stool)

185 variance of persistent phage cluster profiles and 43.9\% (buccal mucosa), $41.9 \%$ (dental

186 plaque), $47.0 \%$ (dorsum of the tongue) and $48.6 \%$ (stool) variance of transient phage cluster

187 profiles can be explained by individual ( $\mathrm{p}<0.001$, PERMANOVA). All viral families are

188 represented in both persistent (Fig. 3e) and transient phage clusters (Fig. 3f). Noticeably, the

189 crAss-like family are prominently represented in persistent phage clusters of stool samples.

190 Both persistent and transient phage clusters are found at various levels of prevalence in these

191 individuals (Fig. 3g). The percentages of persistent phage clusters from one individual also

192 seen in another are 76.0 \pm 21.7 (buccal mucosa), $80.8 \pm 5.7$ (dental plaque), $81.3 \pm 11.1$

193 (dorsum of the tongue) and $75.9 \pm 13.4$ (stool), and for transient clusters are $69.7 \pm 11.0$

194 (buccal mucosa), $83.4 \pm 7.2$ (dental plaque), $81.9 \pm 5.3$ (dorsum of the tongue) and $76.9 \pm 7.1$

195 (stool) (median \pm interquartile range). There are no significant differences in sharing of

196 persistent or transient phage clusters between individuals for each GIT site $(\mathrm{p}=0.477$,

197 Wilcoxon Rank Sum Test).

199 Circular jumbo phages are commonly found in the oral cavity but not in the gut

200 Most phages have a genome size of less than $200 \mathrm{kbp}$, but we found 545 phages with genome

201 sizes greater than $200 \mathrm{kbp}$, known as jumbo phages (Fig. 4a). 366 of these jumbo phages 
202 belong to 109 unique phage clusters, while 179 jumbo phages do not belong to any cluster

203 (Supplementary Table 2). 97 genomes were circularised and are located in oral samples, in

204 particular the dorsum of tongue, but not in stool (Fig. 4b). This is despite the fact that stool

205 samples have the highest proportion of contiguous metagenomic assemblies above $200 \mathrm{kbp}$

206 compared to oral sites (Supplementary Fig. 10). Circular jumbo phage genomes are not

207 present in saliva from the Philippines, but this could be due to fewer assemblies

208 (Supplementary Table 1). The largest circular jumbo phage genomes above $300 \mathrm{kbp}$ are all

209 located in dorsum of tongue samples from the USA (Fig. 4c). Of the six linear megaphage

210 genomes above $500 \mathrm{kbp}$, one is found in a stool sample from China, and the others from three

211 dental plaque and two from dorsum of tongue samples from the USA.

21382 circular jumbo phage genomes are found in 22 phage clusters and the remaining 15 are

214 singletons, meaning 37 distinct circular jumbo phage groupings were identified

215 (Supplementary Table 2). In some cases, both linear and circular jumbo phage genomes are

216 members of the same phage clusters. 29 phage clusters containing 141 jumbo phages are

217 persistent, i.e. found in the same GIT site in more than two timepoints. Ten phage clusters

218 with 87 jumbo phages in the oral cavity are found across more than one country, specifically,

219 four from China, the Philippines and the USA, five from China and the USA, and one from

220 the Philippines and the USA. One phage cluster contains circular and linear jumbo phage

221 genomes that are both persistent and found in all three countries.

223 To investigate whether the genetic variation of these jumbo phages may have arisen from

224 other sources, the phylogeny of their major capsid proteins (MCPs) was compared with those

225 from jumbo phages discovered in metagenomes or isolates from previous studies ${ }^{10,18-20}$.

226 Large phylogenetic radiations of MCPs from oral jumbo phages intersperse with MCPs from 
227 other jumbo phages of human, animal and environmental ecosystems (Fig. 4d). Eight MCPs

228 in jumbo phages identified from the IMG/VR database corroborate with this study's MCPs

229 from the same USA cohort. Notably, jumbo phages from the same clusters and gene content

230 have the most similar MCPs. In one clade, circularised jumbo phages with related MCPs that

231 are from different phage clusters or not belonging to a phage cluster have a conserved

232 syntenic genome structure, but divergent amino acid alignment (Supplementary Fig. 11).

234 Most of the predicted protein-coding genes of these jumbo phages have hypothetical

235 functions, while others mainly encode proteins for replication and nucleic acid metabolism

236 (Fig. 5a, Supplementary Table 3). To investigate the possibility that these jumbo phages may

237 carry ARGs, phage contigs were mapped against the Comprehensive Antibiotic Resistance

238 Database (CARD) ${ }^{22}$. This did not reveal any jumbo phage-associated ARGs. However,

239 auxiliary metabolic genes may provide an alternative pathway to modulating resistance to

240 antimicrobial chemicals. Glutaredoxin, being the third most prevalent auxiliary metabolic

241 gene, is a redox enzyme that catalyses the reduction of disulphide bonds of substrates with

242 cofactor glutathione ${ }^{23}$.

244 Notably, predicted host genera cluster in the same regions as jumbo phages with similar gene

245 profiles (Fig. 5b). Using Random Forest, we identified the most important predictors among

246 host genera by variable importance scores (Permutation Test $\mathrm{p}<0.05$ ). Sets of viral and

247 bacterial proteins are important for an estimated $85.9 \%$ (95\% CI, $77.0-92.3 \%)$ accuracy of

248 host genera (Supplementary Fig. 12). The presence of a CHAP (cysteine, histidine-dependent

249 aminohydrolases/peptidases) domain is an important predictor of multiple hosts, particularly

250 Streptococcus. CHAP domains are usually found as part of peptidoglycan hydrolases ${ }^{25}$. Some 
251 act as endolysins in bacteriophages that digest peptidoglycan of bacterial cell walls. It has

252 been shown in different endolysins that CHAP domains are crucial for lytic activity ${ }^{26,27}$.

\section{Discussion}

255 Bacteriophages are a major but largely neglected component of the oral microbiome. With

256 their ability to introduce and transport genes between strains, as well as modulating

257 composition through lytic activity, they have a significant impact on the population and

258 function of the microbiome as a whole. Recent advances in bioinformatics and high-

259 throughput sequencing now allow us to explore this community. In this study, we defined the

260 composition of the oral phageome and compared it with the gut phageome. Firstly, we

261 showed distinct phageome profiles between sites of the GI tract, particularly between the gut

262 and the oral cavity, with sharing of phage clusters between proximal oral sites. Variations in

263 phageome profiles are likely to be associated with differences in bacterial compositions of

264 these sites that are characteristic of the gut and the oral cavity ${ }^{28}$. It has been observed

265 previously that phages, particularly crAss-like, are both stable and individual-specific in the

266 gut $^{16,29}$. Although we did not find the same levels of crAss-like phages in the oral cavity,

267 other phage clusters can persist for months in different oral sites and are more individual-

268 specific than transient phage clusters. However, we cannot rule out that other less persistent

269 phage clusters in some individuals may be attributed to lower abundance phage contigs not

270 being picked up in our metagenomic pipeline.

271

272 Jumbo phages with circularised genomes were identified in most oral sites, particularly on

273 the dorsum of the tongue from USA samples, but were not found in paired stool samples. The

274 lack of circularised jumbo phages in stool samples is interesting given that stool contains

275 large metagenomic assemblies and a higher diversity of phage clusters than saliva and buccal 
276 mucosa. Circularised jumbo phages have already been discovered in adult faecal samples

277 from the Bangladesh, Tanzania, Peru and the USA ${ }^{18,20}$. However, in a recent study, 19

278 circularised jumbo phages were identified in saliva compared to only one in stool samples

279 from pregnant women in the USA, suggesting the oral cavity may have a naturally higher

280 prevalence of jumbo phages ${ }^{18}$.

282 Recombination of genetic material between bacteriophage genomes is relatively common ${ }^{30}$.

283 This leads to greater variations in synteny (the physical co-localisation of protein-coding

284 genes) particularly for larger bacteriophage genomes, meaning lineage tracing of whole

285 genomes becomes challenging ${ }^{9}$. Previously, it has been shown that large terminase protein

286 and MCP sequences of jumbo phages from environmental, human and animal samples are

287 phylogenetically distinct from those in smaller phages of the same niches, suggesting larger

288 phages may have arisen and evolved alongside smaller ones or that these particular sequences

289 diverged early in evolutionary history ${ }^{18}$. We illustrate that MCPs are relatively conserved

290 with gene content and synteny, which further supports the former idea that jumbo phages

291 across ecological niches may have evolved independently.

293 A jumbo phage's large genome size makes it possible for it to carry a broad range of genes ${ }^{9}$.

294 We find a rich set of both viral and bacterial proteins in jumbo phage genomes of this study.

295 No ARGs were found but instead there was a high prevalence of the auxiliary metabolic gene

296 coding for glutaredoxin. It has been shown that Pseudomonas aeruginosa with mutated

297 monothiol glutaredoxin was more susceptible to polymyxins, a last-line antibiotic for

298 multidrug-resistant bacteria, although the exact mechanism is not known ${ }^{24}$. Bacteriophage

299 genes that are homologous with bacterial genes are likely to have been acquired from host

300 sequences during a previous infection event ${ }^{31}$. Lysogenic phages are also required to adapt 
301 their genetic machinery to integrate and cooperate with the host genome. This may explain

302 why we found a relationship between the incidence of specific jumbo phage proteins and

303 their predicted hosts. Since we could only predict bacterial hosts for $11.8 \%$ of phages

304 (including jumbo phages), functional protein profiles may be able to aid host predictions,

305 especially where CRISPR spacer reference information is lacking. Phage hosts can also be

306 refined more accurately at a metagenomic level using techniques such as SMRT

307 metagenomic sequencing ${ }^{32}$, single-cell viral tagging ${ }^{33}$ or using de novo computational

308 methods ${ }^{31}$.

309

310 The dorsum of the tongue contains the highest diversity of phage clusters compared to other

311 sites, as well as the greatest prevalence of jumbo phages. This could be because bacteria in

312 these sites are largely located in biofilms, which serve to protect microbial communities as

313 well as phages in hostile environments, and bacteriophages themselves have been shown to

314 promote biofilm formation ${ }^{34}$. Dense, protective layers of microbes and their extracellular

315 polymeric substance matrix in biofilms could provide an opportunistic environment for the

316 evolution of disparate phages, including jumbo phages with extended genetic machinery and

317 metabolic capacity ${ }^{9}$.

319 Although we only find circularised jumbo phages in oral sites and a higher diversity of phage

320 clusters on the dorsum of the tongue, we cannot rule out that the gut also may contain

321 comparable levels of diverse phages. It is possible that the total phage composition in the gut

322 could be misrepresented in stool metagenomes. Unlike sampling oral sites of particular

323 physical locations, such as dental plaque, surface of the tongue and mucosa from the inner

324 cheek, sampling faecal matter is unable to capture the spatial microbial community structure

325 of the gut, both radially and longitudinally ${ }^{35}$. The microbial community structure of the 
326 human gut is of considerable importance to the dynamics of bacteriophage populations.

327 Studies applying transmission electron microscopy of human gut biopsies have shown higher

328 levels of bacteriophage colonisation in the colonic mucosa layer ${ }^{36}$ than in faeces and

329 caecum $^{37}$. Several existing models (piggyback-the-winner ${ }^{38}$ and kill-the-winner ${ }^{39}$ ) have been

330 proposed to explain this biogeographical variation in bacteriophage density across the radial

331 direction in the gut ${ }^{40}$. Lysogeny dominates in low virus to microbe ratios in the gut lumen by

332 the piggyback-the-winner model, but switches to the lytic cycle in higher microbial densities

333 of the mucin layer by the kill-the-winner model.

335 Already, significant contributions have been made in the discovery of multitudes of novel

336 bacteriophage genomes in humans, animals, outdoor and contained environments ${ }^{8,10,18,20}$.

337 However, further investigations into how genetic and functional variations are manifested at

338 more specific biogeographical sites are required. With better structural resolution, it will be

339 possible to capture the extent of genetic diversity and profile the dynamics of distinct phages

340 in different human microbiomes, such as biofilms on the surface of the tongue. Clinically,

341 there has been renewed interest in using phage therapy with other antimicrobial therapies to

342 control biofilms ${ }^{34}$. In the future, combinations of genotypic attributes that influence phage

343 persistence and interactions with their hosts could be used to select or design phages for

344 eradicating chronic infections, like periodontitis and dental caries caused by consortia of

345 organisms in biofilms ${ }^{41}$.

347 Methods

348 Metagenomic data for creating the phage catalogue

349 A total of 1061 publicly available metagenomic samples covering the USA, China and the

350 Philippines, all sequenced using Illumina HiSeq 2000, were used to create a reference phage 
351 contig catalogue. Longitudinal USA samples were excluded from the majority of the study

352 after the first timepoint to ensure each sample was independent, unless specified otherwise.

353 All metagenomes passed over half the quality control metrics in FastQC 0.11.3

354 (https://www.bioinformatics.babraham.ac.uk/projects/fastqc/) with these pass rates calculated

355 in MultiQC ${ }^{42}$. These samples include 1) longitudinal data across two years with various

356 timepoints from the Human Microbiome Project 1 (referred to as USA) containing buccal

357 mucosa ( $\mathrm{n}=87: 32$ with one, 35 with two, 19 with three and 1 with six timepoints); dorsum

358 of tongue ( $\mathrm{n}=91: 22$ with one, 43 with two, 24 with three and 2 with four timepoints); dental

359 plaque ( $\mathrm{n}=90: 24$ with one, 41 with two, 21 with three, 1 with four and 3 with six

360 timepoints); stool ( $\mathrm{n}=70: 13$ with one, 33 with two, 21 with three, 2 with four and 1 with six

361 timepoints $^{43}, 2$ ) healthy controls and rheumatoid arthritis (RA) patients from a Chinese study

362 containing dental plaque (healthy: $\mathrm{n}=32$, RA: $\mathrm{n}=76$ ); saliva (healthy: $\mathrm{n}=33$, RA: $\mathrm{n}=24$ );

363 stool (healthy: $\mathrm{n}=72$, RA: $\mathrm{n}=100)^{44}$, and 3 ) saliva samples $(\mathrm{n}=24)$ from healthy hunter-

364 gatherers and traditional farmers from the Philippines ${ }^{45}$.

366 Raw paired-end metagenomic reads from Chinese and Philippines samples were downloaded

367 from the EBI (https://www.ebi.ac.uk/metagenomics/). Paired-end metagenomic samples from

368 USA were downloaded from https://portal.hmpdacc.org/. All USA, China and Philippines

369 samples were collected and sequenced as described in the following cited studies ${ }^{43-45}$.

\section{Processing metagenomic data}

372 The raw reads for all samples were trimmed using AlienTrimmer v0.4. $0^{46}$ with parameters $-k$

$373 \quad 10-l 45-m 5-p 40-q 20$ and Illumina contaminant oligonucleotides

374 (https://gitlab.pasteur.fr/aghozlan/shaman_bioblend/blob/18a17dbb44cece4a8320cce8184adb

375 9966583aaa/alienTrimmerPF8contaminants.fasta). Human contaminant sequences were 
376 removed from all samples by discarding reads that mapped against a human reference

377 genome (downloaded from Human Genome Resources at NCBI on $27^{\text {th }}$ February 2017) using

378 Bowtie 2 v2.2.3 $3^{47}$ with parameters $-q-N 1$ - $k 1$--fr --end-to-end --phred33 --very-sensitive --

379 no-discordant.

\section{Phage contig catalogue}

382 The reads were assembled into contigs using SPAdes v3.9.0 $0^{48}$ with parameters $-k 21,33,55$--

383 only-assembler --meta. Small contigs with a length of less than 3000 bp were removed.

384 Linear and circular phage contigs were identified by searching for viral signatures using

385 VirSorter v1.0.5 ${ }^{14}$. The phage contigs were clustered with multi-alignment using BLASTn

386 v2.6.0 ${ }^{49}$ with parameters -evalue 1e-20 -word_size 100 -max_target_seqs 10000 and

387 redundant contigs were removed where identity and breadth coverage were both greater than

388 and equal to $90 \%$. Phage contigs were then clustered into viral clusters using vConTACT2

389 v0.9.10 with all default parameters ${ }^{15}$. Taxonomic classification of phage contigs to Order and

390 Family level was predicted using Demovir (build 20 ${ }^{\text {th }}$ April 2018) on phage proteins with an

391 e-value cut-off of 1e-5 (https://github.com/feargalr/Demovir). Non-phage viral families,

392 Alloherpesviridae, Ascoviridae, Baculoviridae, Flaviviridae, Herpesviridae, Iridoviridae,

393 Marseilleviridae, Mimiviridae, Nudiviridae, Phycodnaviridae, Picornaviridae, Pithoviridae,

394 Poxviridae and Retroviridae, were labelled as "Other". Although some phage clusters were

395 labelled as non-phage, the developers of Demovir recommend against using their

396 classification to discriminate between bacterial, archaeal and eukaryotic sequences from

397 metagenomic samples. The phage hosts were predicted by aligning phage contigs against a

398 database of CRISPR spacers as described by Shkoporov et al., 2019 ${ }^{16}$. Metadata for the phage

399 catalogue were assembled using the helper script "create_catalogue_dataset.R". 


\section{Sequence and functional annotation of jumbo phages}

402 Circular phage genomes of length $>200 \mathrm{kbp}$ and linear phage genomes of length $>200 \mathrm{kbp}$

403 that were connected to these circular phages in vConTACT2's gene-sharing network were put

404 forward as candidate jumbo phages using the helper script "get_candidate_jumbophages.R".

405 The scaffold file containing their genomes was generated using the helper script

406 “extract_jumbophage_contigs.py".

407

408 The candidate jumbo phage genomes were annotated for functional proteins and tRNA genes.

409 Protein prediction was conducted using Prodigal v2.6.3 ${ }^{50}$ with parameters - $p$ meta. Protein

410 sequences were searched against databases of Hidden Markov models, prokaryotic virus

411 orthologous groups (pVOGs) (downloaded $1^{\text {st }}$ November 2019$)^{51}, \mathrm{pFAMs}^{52}\left(\right.$ downloaded $2^{\text {nd }}$

412 September 2019) and TIGRFAMs ${ }^{53}$ (downloaded $3^{\text {rd }}$ September 2019), using hmmsearch

413 v3.2.1 $1^{54}$ with e-value cut-off of 1e-5. tRNA genes were identified from nucleotide sequences

414 using ARAGORN v1.2.36 with parameters $-t-i-c-d-w$ and with helper script

415 “clean_aragorn_output.py”. The hit with the lowest e-value and domain e-value was selected

416 from for every query protein with candidate target protein hits for each database. Next, the hit

417 with the highest bit score and domain bit score was selected for every query protein with

418 candidate target protein hits from more than one database. The few remaining protein query

419 sequences with the same e-values and hit scores were deduplicated. These steps were run

420 using the helper script "collate_functional_annotations.R". Circular candidate jumbo phages

$421>200 \mathrm{kbp}$ that did not contain a major capsid protein (MCP) were also excluded, leaving a

422 total of 545 putative jumbo phages.

\section{Phage annotation in metagenomes}


425854 metagenomes from healthy individuals were mapped against the non-redundant phage

426 catalogue using Bowtie2 v2.3.4.1. Phage contigs (excluding spurious jumbo phages) and

427 phage clusters were quantified for each sample where contig breadth coverage was $75 \%$ or

428 greater using helper script "phage_quantification.R”. Relative phage abundance profiles were

429 calculated by scaling the depth coverage of phage contigs that were divided by total reads per

430 sample. The metagenomes came from the USA with buccal mucosa $(n=87: 32$ with one, 35

431 with two, 19 with three and 1 with six timepoints); dorsum of tongue ( $\mathrm{n}=90: 22$ with one, 43

432 with two, 24 with three and 2 with four timepoints); dental plaque ( $\mathrm{n}=90: 24$ with one, 41

433 with two, 21 with three, 1 with four and 3 with six timepoints); and stool samples ( $\mathrm{n}=70: 13$

434 with one, 33 with two, 21 with three, 2 with four and 1 with six timepoints), China with

435 dental plaque $(\mathrm{n}=32)$; saliva $(\mathrm{n}=33)$; and stool samples $(\mathrm{n}=72)$, and the Philippines with

436 saliva samples $(n=24)$. Metadata for the samples can be found in Supplementary Table 1.

437 The following analysis was conducted in script "phage_analysis.R".

439 Phage diversity

440 To find differences in beta-diversity of phage profiles between groups of individuals, the

441 Bray-Curtis dissimilarity of phage incidence (presence or absence) profiles was computed

442 between individuals and visualised using NMDS. Silhouette analysis of k-medoids was used

443 to select the number of distinct groups with the largest Silhouette width.

445 The alpha-diversity was calculated as the phage cluster richness which is the number of

446 unique phage clusters for each sample. Only samples with greater than 100 phages were

447 included. Individuals with samples containing less than or equal to three clusters were

448 excluded from the group comparison. The phage cluster richness was compared between

449 paired GIT sites from the same individuals in each of the following groups: China dental 
450 plaque vs. saliva $(\mathrm{n}=30)$; China stool vs. saliva $(\mathrm{n}=30)$; China stool vs. dental plaque $(\mathrm{n}=$

$45130)$; USA buccal mucosa vs. dental plaque $(n=45)$; USA buccal mucosa vs. dorsum of

452 tongue $(n=45)$; USA buccal mucosa vs. stool $(n=36)$; USA dental plaque vs. dorsum of

453 tongue $(\mathrm{n}=86)$; USA dental plaque vs. stool $(\mathrm{n}=67)$; and USA dorsum of tongue vs. stool ( $\mathrm{n}$

$454=68$ ). Since the number of phage contigs in each sample is significantly linearly correlated

455 with Phage Cluster Richness ( $\mathrm{p}<2.2 \times 10^{-16}$ ) (Supplementary Fig. 13), the number of phage

456 clusters for each sample were subsampled to the smallest number of phages found in a

457 sample for each paired comparison. The phage cluster richness between samples in each

458 group was tested for statistical significance with a Two-sided Wilcoxon Rank Sum Test.

\section{Microbial composition}

461 MetaPhlAn2 v2.6.0 $0^{17}$ was used to identify the composition of bacteria and archaea from

462 samples apart from longitudinal USA samples. One dorsum of tongue USA sample did not

463 have bacterial nor archaeal microbial predictions. Procrustes analysis was applied to visualise

464 the superposition of NMDS dimensions of phage incidence profiles on microbial genera

465 incidence profiles using the protest function in the vegan package v2.5.6 in R. PROTEST was

466 performed with 999 permutations to a significance of $\mathrm{p}=0.001$.

\section{Longitudinal analysis of phages}

469 The stability of phages was investigated by computing the number of timepoints each phage

470 cluster is found from each individual and GIT site in the longitudinal USA data. The

471 proportion of phage clusters and reads mapped against these clusters were calculated for each

472 number of sampled timepoints available for each individual and GIT site: buccal mucosa $(\mathrm{n}=$

$47320)$, dental plaque $(n=24)$, dorsum of the tongue $(n=26)$ and stool $(n=24)$. Persistent phage

474 clusters are defined as being found in three or more timepoints, whereas transient phage 
475 clusters are defined as being found in less than three timepoints. The Bray-Curtis

476 dissimilarity was computed between persistent and transient phage cluster incidence profiles

477 from GIT sites of individuals containing both persistent and transient phage clusters: buccal

478 mucosa $(n=18)$, dental plaque $(n=23)$, dorsum of the tongue $(n=25)$ and stool $(n=24)$.

479 Non-metric multidimensional scaling was applied to scale the dissimilarity into a two-

480 dimensional ordination using the metaMDS function in the vegan package v2.5.6 in R.

481 PERMANOVA analysis was performed using the adonis function in the vegan package.

\section{Antimicrobial resistance gene annotation of jumbo phages}

484 Phage contigs were annotated for ARGs by mapping against CARD v3.0.0 using BLASTn

485 v2.10.0 with parameters -evalue 1e-5. Hits were filtered by $90 \%$ identity.

\section{Phylogenetic analysis of MCPs}

488 A phylogenetic tree was constructed from MCP sequences of jumbo phages identified from this study, complete dsDNA jumbo phage genomes from RefSeq $\mathrm{r} 99^{19}$, and jumbo phage scaffolds from IMG/VR ${ }^{56}$, Al-Shayeb et al. ${ }^{18}$ and Devoto et al. ${ }^{10}$. MCPs from IMG/VR jumbo

491 phage scaffolds were identified using the functional annotation pipeline described above.

492 Jumbo phages from Al-Shayeb et al. that contain MCPs were identified using the helper 493 script "get_files_with_MCPs.sh" from supplementary Genbank files, generated as described

494 in Al-Shayeb et al. ${ }^{18}$. MCPs of complete jumbo phages were also collected from Genbank

495 files downloaded from RefSeq r99. All MCPs were collated into a file of amino acid 496 sequences using the helper script “get_MCPs.py”. The MCP amino acid sequences were 497 clustered using CD-HIT ${ }^{57}$ at $100 \%$ identity to remove redundancy. The output sequences 498 were aligned using MAFFT $\mathrm{v} 7.453^{58}$ using parameters --localpair --maxiterate 1000. The 499 phylogenetic tree was constructed using IQTREE v1.6.12 ${ }^{59}$ with automatic model selection 
500 and 1000 bootstrap replicates, and visualised using iTOL v5.5.1 ${ }^{60}$ with the tree rooted at

501 midpoint. Metadata of these jumbo phages for the tree was created using helper scripts

502 “get_RefSeq_info.py”, “create_tree_metadata.R” and

503 “add_metadata_to_tree_annotations.sh".

504

505 Synteny comparison

506 Pairwise alignments of genomes of four circular jumbo phages with closely related MCPs

507 (SRS078425_NODE_1_length_226348_cov_18.355,

508 SRS019607_NODE_9_length_235695_cov_219.599,

509 ERR589420_NODE_6_length_229992_cov_13.7426,

510 SRS044662_NODE_25_length_232905_cov_15.7753) were performed using tblastx v2.10.0

511 within Easyfig v2.2.2 $2^{61}$. Genbank files of the circular jumbo phages were generated using

512 RASTtk v1.3.0 $0^{62}$ and separate genbank files were created for the four jumbo phages using the

513 helper script "extract_genbank_files.py". MCPs with type "CDS" in their Genbank files were

514 relabelled "MCP". Genomes were linearised and fixed by MCP as the starting protein-coding

515 gene using the helper script "reorder_genbank_files.py".

\section{Phage Host Prediction}

518 A Random Forest classification model was created to analyse the relationship between

519 functional profiles in jumbo phages and their host genera. Incidence of protein function for

520109 phage clusters containing 366 jumbo phages and 179 jumbo phages with no assigned

521 phage cluster, that have CRISPR spacer-predicted hosts, were used to train the model using

522 the randomForest package v4.6-14 in R. The accuracy of the trained model was evaluated

523 using the in-built out-of-bag error estimation. The Mean Decrease in Accuracy was

524 determined to measure the importance of protein functions for predicting host genera. Its 
525 significance was estimated by permuting the host genera response variable to produce null

526 distributions of the Mean Decrease in Accuracy (scaled by the standard error) for each

527 protein function predictor using the rfPermute package v2.1.81.

528

529 Code availability

530 The code for the analysis is available from https://github.com/APC-Microbiome-

531 Ireland/phageome_analysis

532

533 References

534 1. Fernández, L., Rodríguez, A. \& García, P. Phage or foe: an insight into the impact of viral

535 predation on microbial communities. ISME J. 1 (2018) doi:10.1038/s41396-018-0049-5.

536 2. Hsu, B. B. et al. Dynamic Modulation of the Gut Microbiota and Metabolome by

537 Bacteriophages in a Mouse Model. Cell Host Microbe 25, 803-814.e5 (2019).

538 3. Clooney, A. G. et al. Whole-Virome Analysis Sheds Light on Viral Dark Matter in

539 Inflammatory Bowel Disease. Cell Host Microbe 26, 764-778.e5 (2019).

540 4. Shkoporov, A. N. et al. Reproducible protocols for metagenomic analysis of human

541 faecal phageomes. Microbiome 6, 68 (2018).

542 5. Roux, S. et al. Minimum Information about an Uncultivated Virus Genome (MIUViG).

$543 \quad$ Nat. Biotechnol. (2018) doi:10.1038/nbt.4306.

544 6. Koonin, E. V. \& Yutin, N. The crAss-like Phage Group: How Metagenomics Reshaped the

$545 \quad$ Human Virome. Trends Microbiol. 28, 349-359 (2020).

546 7. Yutin, N. et al. Discovery of an expansive bacteriophage family that includes the most

547 abundant viruses from the human gut. Nat. Microbiol. 3, 38-46 (2018). 
548 8. Edwards, R. A. et al. Global phylogeography and ancient evolution of the widespread

549 human gut virus crAssphage. Nat. Microbiol. 4, 1727-1736 (2019).

550 9. Yuan, Y. \& Gao, M. Jumbo Bacteriophages: An Overview. Front. Microbiol. 8, (2017).

551 10. Devoto, A. E. et al. Megaphages infect Prevotella and variants are widespread in gut

552 microbiomes. Nat. Microbiol. 1 (2019) doi:10.1038/s41564-018-0338-9.

553 11. Pride, D. T. et al. Evidence of a robust resident bacteriophage population revealed

554 through analysis of the human salivary virome. ISME J. 6, 915-926 (2012).

555 12. Wang, J., Gao, Y. \& Zhao, F. Phage-bacteria interaction network in human oral

556 microbiome. Environ. Microbiol. 18, 2143-2158 (2016).

557 13. Hayes, S., Mahony, J., Nauta, A. \& van Sinderen, D. Metagenomic Approaches to Assess

$558 \quad$ Bacteriophages in Various Environmental Niches. Viruses 9, 127 (2017).

559 14. Roux, S., Enault, F., Hurwitz, B. L. \& Sullivan, M. B. VirSorter: mining viral signal from

560 microbial genomic data. PeerJ 3, e985 (2015).

561 15. Jang, H. B. et al. Taxonomic assignment of uncultivated prokaryotic virus genomes is

562 enabled by gene-sharing networks. Nat. Biotechnol. 37, 632-639 (2019).

563 16. Shkoporov, A. N. et al. The Human Gut Virome Is Highly Diverse, Stable, and Individual

$564 \quad$ Specific. Cell Host Microbe 26, 527-541.e5 (2019).

565 17. Truong, D. T. et al. MetaPhIAn2 for enhanced metagenomic taxonomic profiling. Nat.

$566 \quad$ Methods 12, 902-903 (2015).

567 18. Al-Shayeb, B. et al. Clades of huge phages from across Earth's ecosystems. Nature 1-7

$568 \quad$ (2020) doi:10.1038/s41586-020-2007-4.

569 19. O'Leary, N. A. et al. Reference sequence (RefSeq) database at NCBI: current status,

570 taxonomic expansion, and functional annotation. Nucleic Acids Res. 44, D733-745

$571 \quad$ (2016). 
572 20. Paez-Espino, D. et al. Uncovering Earth's virome. Nature 536, 425-430 (2016).

573 21. Enault, F. et al. Phages rarely encode antibiotic resistance genes: a cautionary tale for

$574 \quad$ virome analyses. ISME J. 11, 237-247 (2017).

575 22. McArthur, A. G. et al. The comprehensive antibiotic resistance database. Antimicrob.

$576 \quad$ Agents Chemother. 57, 3348-3357 (2013).

577 23. Fernandes, A. P. \& Holmgren, A. Glutaredoxins: Glutathione-Dependent Redox Enzymes

578 with Functions Far Beyond a Simple Thioredoxin Backup System. Antioxid. Redox Signal.

$5796,63-74(2004)$.

580 24. Romsang, A., Leesukon, P., Duangnkern, J., Vattanaviboon, P. \& Mongkolsuk, S.

$581 \quad$ Mutation of the gene encoding monothiol glutaredoxin (GrxD) in Pseudomonas

582 aeruginosa increases its susceptibility to polymyxins. Int. J. Antimicrob. Agents 45, 314-

$583 \quad 318(2015)$.

584 25. Rigden, D. J., Jedrzejas, M. J. \& Galperin, M. Y. Amidase domains from bacterial and

585 phage autolysins define a family of $y$-d, l-glutamate-specific amidohydrolases. Trends

$586 \quad$ Biochem. Sci. 28, 230-234 (2003).

587 26. Horgan, M. et al. Phage Lysin LysK Can Be Truncated to Its CHAP Domain and Retain

588 Lytic Activity against Live Antibiotic-Resistant Staphylococci. Appl. Environ. Microbiol. 75,

$589 \quad 872-874(2009)$.

590 27. Becker, S. C. et al. Lytic activity of the staphylolytic Twort phage endolysin CHAP domain 591 is enhanced by the SH3b cell wall binding domain. FEMS Microbiol. Lett. 362, 1-8 (2015).

592 28. Segata, N. et al. Composition of the adult digestive tract bacterial microbiome based on 593 seven mouth surfaces, tonsils, throat and stool samples. Genome Biol. 13, R42 (2012).

594 29. Shkoporov, A. N. et al. ФCrAss001 represents the most abundant bacteriophage family 595 in the human gut and infects Bacteroides intestinalis. Nat. Commun. 9, 4781 (2018). 
596 30. Mavrich, T. N. \& Hatfull, G. F. Bacteriophage evolution differs by host, lifestyle and

597 genome. Nat. Microbiol. 2, 1-9 (2017).

598 31. Edwards, R. A., McNair, K., Faust, K., Raes, J. \& Dutilh, B. E. Computational approaches to 599 predict bacteriophage-host relationships. FEMS Microbiol. Rev. 40, 258-272 (2016).

600 32. Beaulaurier, J. et al. Metagenomic binning and association of plasmids with bacterial

601 host genomes using DNA methylation. Nat. Biotechnol. 36, 61-69 (2018).

602 33. Džunková, M. et al. Defining the human gut host-phage network through single-cell viral 603 tagging. Nat. Microbiol. 1-12 (2019) doi:10.1038/s41564-019-0526-2.

604 34. Hansen, M. F., Svenningsen, S. L., Røder, H. L., Middelboe, M. \& Burmølle, M. Big Impact 605 of the Tiny: Bacteriophage-Bacteria Interactions in Biofilms. Trends Microbiol. 0, (2019).

606 35. Allaband, C. et al. Microbiome 101: Studying, Analyzing, and Interpreting Gut

607 Microbiome Data for Clinicians. Clin. Gastroenterol. Hepatol. Off. Clin. Pract. J. Am.

608 Gastroenterol. Assoc. 17, 218-230 (2019).

609 36. Lepage, P. et al. Dysbiosis in inflammatory bowel disease: a role for bacteriophages? Gut $610 \quad 57,424-425(2008)$.

611 37. Hoyles, L. et al. Characterization of virus-like particles associated with the human faecal 612 and caecal microbiota. Res. Microbiol. 165, 803-812 (2014).

613 38. Knowles, B. et al. Lytic to temperate switching of viral communities. Nature 531, 466$614 \quad 470(2016)$.

615 39. Thingstad, T. F. Elements of a theory for the mechanisms controlling abundance, 616 diversity, and biogeochemical role of lytic bacterial viruses in aquatic systems. Limnol. 617 Oceanogr. 45, 1320-1328 (2000).

618 40. Shkoporov, A. N. \& Hill, C. Bacteriophages of the Human Gut: The "Known Unknown" of 619 the Microbiome. Cell Host Microbe 25, 195-209 (2019). 
620 41. Jenkinson, H. F. \& Lamont, R. J. Oral microbial communities in sickness and in health.

621 Trends Microbiol. 13, 589-595 (2005).

622 42. Ewels, P., Magnusson, M., Lundin, S. \& Käller, M. MultiQC: summarize analysis results

623 for multiple tools and samples in a single report. Bioinforma. Oxf. Engl. 32, 3047-3048

$624 \quad$ (2016).

625 43. Human Microbiome Project Consortium. A framework for human microbiome research.

$626 \quad$ Nature 486, 215-221 (2012).

627 44. Zhang, X. et al. The oral and gut microbiomes are perturbed in rheumatoid arthritis and

628 partly normalized after treatment. Nat. Med. 21, 895-905 (2015).

629 45. Lassalle, F. et al. Oral microbiomes from hunter-gatherers and traditional farmers reveal

630 shifts in commensal balance and pathogen load linked to diet. Mol. Ecol. 27, 182-195

$631 \quad$ (2018).

632 46. Criscuolo, A. \& Brisse, S. AlienTrimmer: A tool to quickly and accurately trim off multiple

633 short contaminant sequences from high-throughput sequencing reads. Genomics 102,

$634 \quad 500-506(2013)$.

635 47. Langmead, B. \& Salzberg, S. L. Fast gapped-read alignment with Bowtie 2. Nat. Methods

6369 9, 357-359 (2012).

637 48. Bankevich, A. et al. SPAdes: A New Genome Assembly Algorithm and Its Applications to

638 Single-Cell Sequencing. J. Comput. Biol. 19, 455-477 (2012).

639 49. Altschul, S. F., Gish, W., Miller, W., Myers, E. W. \& Lipman, D. J. Basic local alignment

640 search tool. J. Mol. Biol. 215, 403-410 (1990).

641 50. Hyatt, D. et al. Prodigal: prokaryotic gene recognition and translation initiation site

642 identification. BMC Bioinformatics 11, 119 (2010). 
643 51. Grazziotin, A. L., Koonin, E. V. \& Kristensen, D. M. Prokaryotic Virus Orthologous Groups

644 (pVOGs): a resource for comparative genomics and protein family annotation. Nucleic

$645 \quad$ Acids Res. 45, D491-D498 (2017).

646 52. Finn, R. D. et al. The Pfam protein families database. Nucleic Acids Res. 36, D281-D288

$647 \quad$ (2008).

648 53. Haft, D. H., Selengut, J. D. \& White, O. The TIGRFAMs database of protein families.

$649 \quad$ Nucleic Acids Res. 31, 371-373 (2003).

650 54. Eddy, S. R. Accelerated Profile HMM Searches. PLOS Comput. Biol. 7, e1002195 (2011).

651 55. Laslett, D. \& Canback, B. ARAGORN, a program to detect tRNA genes and tmRNA genes

652 in nucleotide sequences. Nucleic Acids Res. 32, 11-16 (2004).

653 56. Paez-Espino, D. et al. IMG/VR v.2.0: an integrated data management and analysis

654 system for cultivated and environmental viral genomes. Nucleic Acids Res. 47, D678-

655 D686 (2019).

656 57. Fu, L., Niu, B., Zhu, Z., Wu, S. \& Li, W. CD-HIT: accelerated for clustering the next-

657 generation sequencing data. Bioinformatics 28, 3150-3152 (2012).

658 58. Katoh, K. \& Standley, D. M. MAFFT Multiple Sequence Alignment Software Version 7:

659 Improvements in Performance and Usability. Mol. Biol. Evol. 30, 772-780 (2013).

660 59. Trifinopoulos, J., Nguyen, L.-T., von Haeseler, A. \& Minh, B. Q. W-IQ-TREE: a fast online

661 phylogenetic tool for maximum likelihood analysis. Nucleic Acids Res. 44, W232-W235

662 (2016).

663 60. Letunic, I. \& Bork, P. Interactive Tree Of Life (iTOL) v4: recent updates and new

664 developments. Nucleic Acids Res. 47, W256-W259 (2019).

665 61. Sullivan, M. J., Petty, N. K. \& Beatson, S. A. Easyfig: a genome comparison visualizer.

666 Bioinforma. Oxf. Engl. 27, 1009-1010 (2011). 
667 62. Brettin, T. et al. RASTtk: a modular and extensible implementation of the RAST

668 algorithm for building custom annotation pipelines and annotating batches of genomes.

669 Sci. Rep. 5, 8365 (2015).

670 63. Rams, T. E., Hawley, C. E., Whitaker, E. J., Degener, J. E. \& van Winkelhoff, A. J.

671 Centipeda periodontii in human periodontitis. Odontology 103, 286-291 (2015).

673 Acknowledgements

674 The research was supported by the Centre for Host-Microbiome Interactions, King's College

675 London, funded by the Biotechnology and Biological Sciences Research Council (BBSRC)

676 grant BB/M009513/1 awarded to D.L.M., The Alan Turing Institute under the Engineering

677 and Physical Sciences Research Council (EPSRC) grant EP/N510129/1, and APC

678 Microbiome Ireland funded by the Research Centre grant from Science Foundation Ireland

679 (SFI) under Grant Number SFI/12/RC/2273. The authors would like to thank Professor

680 Gordon B. Proctor, Dr Saeed Shoaie and Dr Sunjae Lee for constructive criticism of the

681 manuscript.

682

683 Author contributions

684 V.R.C. and C.H. conceived the presented idea. A.S. created the phage catalogue. A.S. and

685 V.R.C. did the data analysis. V.R.C. and A.S. wrote the manuscript with support from C.H.,

686 D.L.M., D.G-C and P.M.

687

688 Conflicts of interest

689 The authors declare no conflicts of interest. 
a

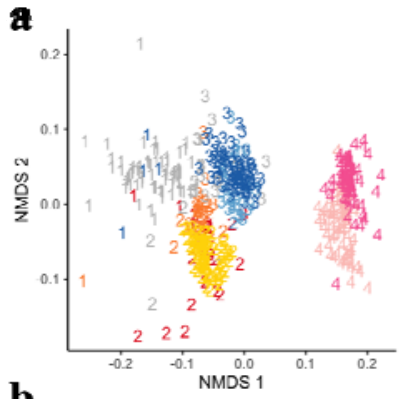

b

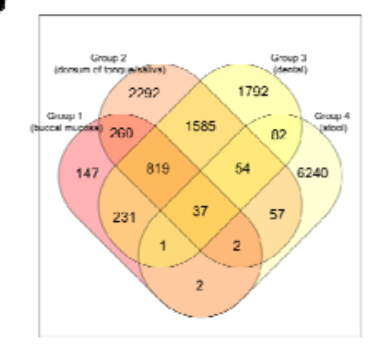

c

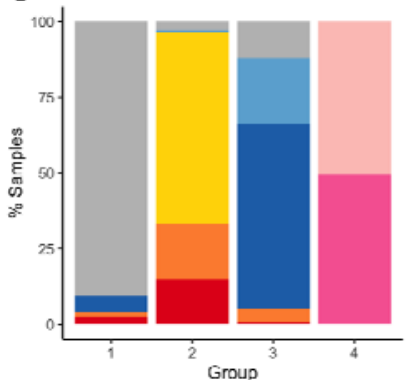

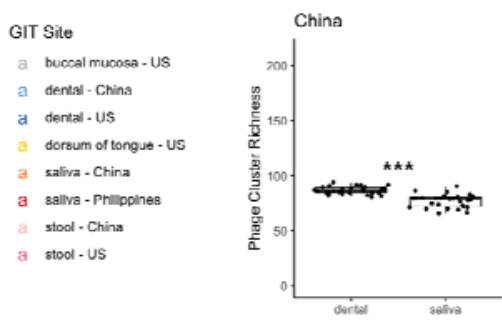
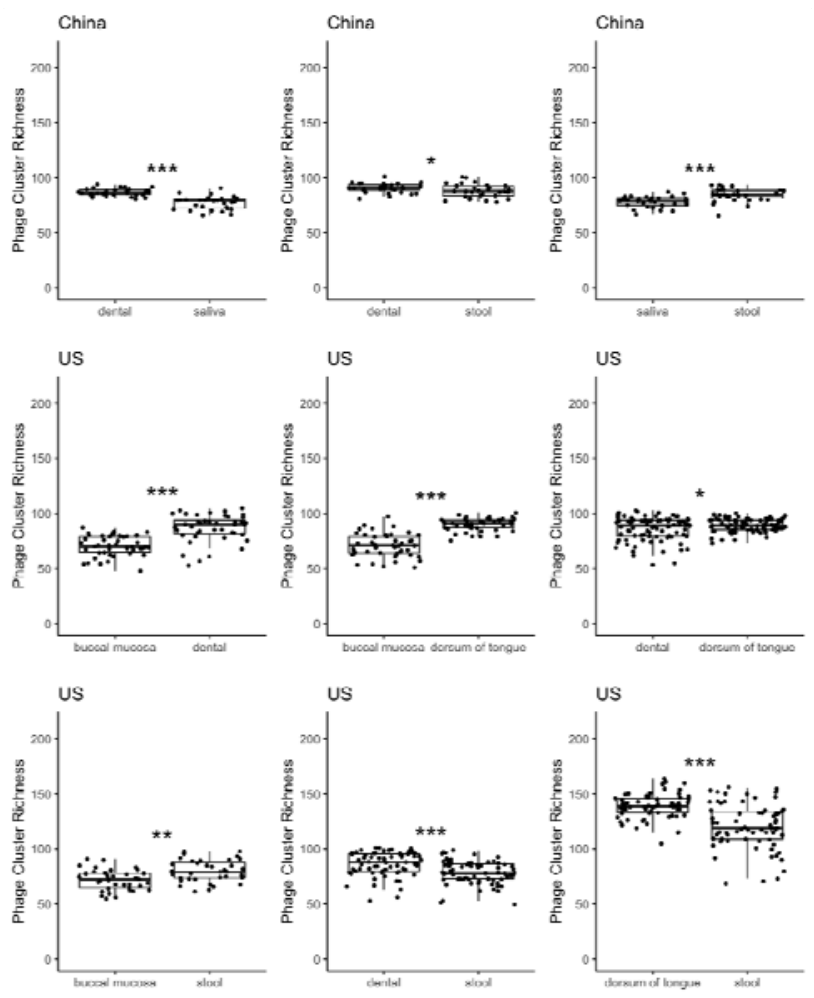

Figure 1: Phage incidence and abundance profiles. a) Non-metric multidimensional scaling of Bray-Curtis dissimilarity between phage incidence profiles of samples (excluding longitudinal USA). Ordination coordinates are grouped by k-medoids clustering, where number of groups, $k$, has the largest Silhouette width. USA buccal mucosa $(\mathrm{n}=87)$, dorsum of tongue $(\mathrm{n}=90)$, dental plaque $(\mathrm{n}=90)$ and stool $(\mathrm{n}=70)$; China dental plaque $(\mathrm{n}=32)$, saliva $(\mathrm{n}=33)$ and stool $(\mathrm{n}=72)$; and Philippines saliva $(\mathrm{n}=24)$. b) Number of viral clusters in each group. c) Percentage of samples in each group from 1a, labelled by GIT site and country. d) Phage Cluster Richness between paired GIT sites. Phage Cluster Richness is defined as the number of unique viral clusters in a sample that is subsampled to the smallest number of non-unique clusters. Phage Cluster Richness is calculated for samples of individuals from China (dental plaque and saliva: $\mathrm{n}=30$, stool and saliva: $\mathrm{n}=30$, stool and dental plaque: $n=30$ ) and the USA (buccal mucosa and dental plaque: $n=45$, buccal mucosa and dorsum of tongue: $n=45$, buccal mucosa and stool: $n=36$, dental plaque and dorsum of tongue: $n=86$, dental plaque and stool: $n=67$, dorsum of tongue and stool: $n=68)$ (excluding longitudinal USA) with Two-sided Wilcoxon Rank Sum Test ( $\mathrm{p}<0.05$ as *, < 0.01 as $* *,<0.005$ as $* * *)$. Centre line is median, box limits are upper and lower quartiles, whiskers are $1,5 \mathrm{x}$ interquartile ranges and points beyond whiskers are outliers. 
711

712

713

714

715

716

717

718

719

720

721

722

723

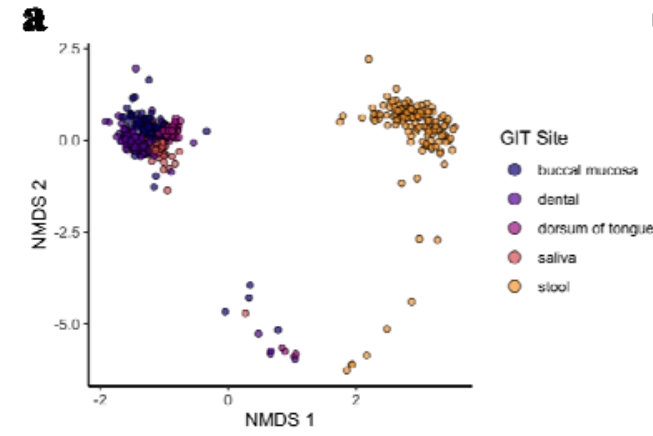

b

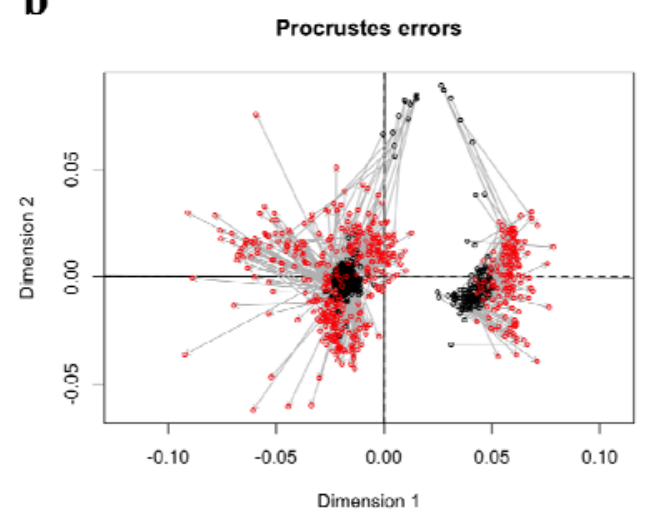

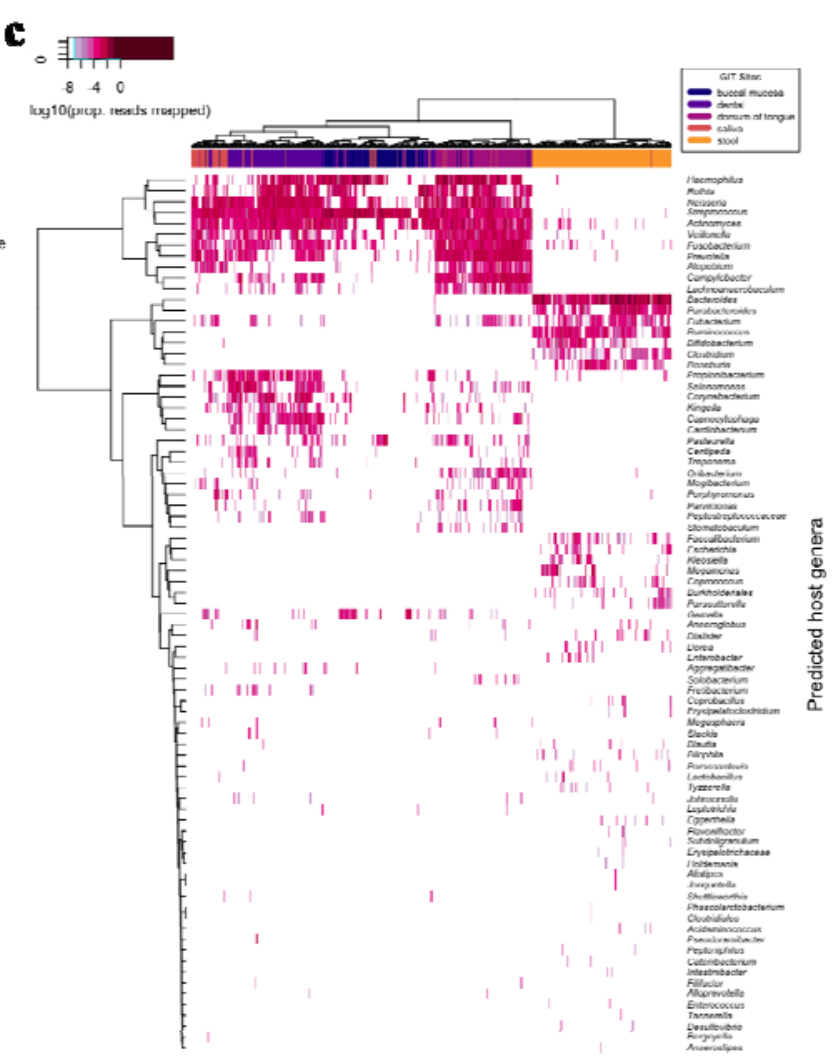

Figure 2: Relationship between phage profiles and microbial composition, and abundance of predicted phage hosts. a) Non-metric multidimensional scaling (NMDS) of Bray-Curtis dissimilarity between microbial taxa incidence of samples (excluding longitudinal USA and $\mathrm{n}=21$ samples with suspected contamination) labelled by GIT site. b) Procrustes rotation of NMDS coordinates between microbial genera profile from 2a (black) and phageome profile from 1a (red). Correlation in symmetric Procrustes rotation $=0.70(\mathrm{p}=$ $0.001 ; 999$ permutations; PROTEST). c) $\log 10$ of the proportion of reads mapped to phage contigs with predicted host for each sample, clustered by hierarchical clustering, $\mathrm{x}$-axis coloured by GIT site and $\mathrm{y}$-axis labelled by genus of predicted host. USA buccal mucosa $(\mathrm{n}=$ 87), dorsum of tongue $(\mathrm{n}=90)$, dental plaque $(\mathrm{n}=90)$ and stool $(\mathrm{n}=70)$; China dental plaque $(\mathrm{n}=32)$, saliva $(\mathrm{n}=33)$ and stool $(\mathrm{n}=72)$; and Philippines saliva $(\mathrm{n}=24)$. 

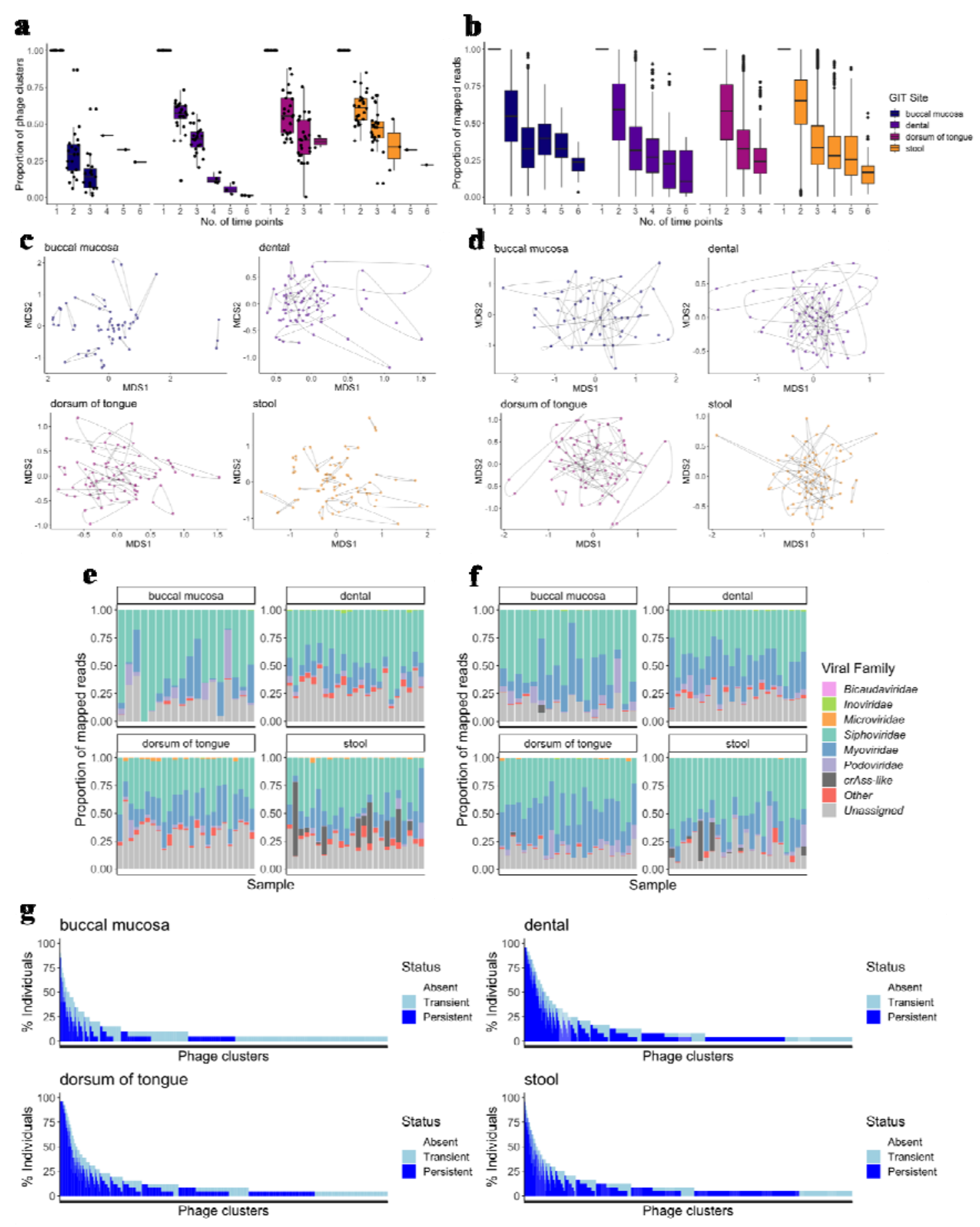

725 Figure 3: Phage cluster stability in longitudinal USA oral and gut samples. Proportion of

726 a) phage clusters and b) reads mapped to phage clusters, in one to six timepoints for USA individuals with at least three sampling timepoints (buccal mucosa: $n=20$, dental plaque: $n=$ 24 , dorsum of the tongue: $n=26$, stool: $n=24$ ). Non-metric multidimensional scaling of the Bray-Curtis dissimilarity between phage clusters incidence profiles of samples with c) persistent and d) transient phage clusters. Points represent samples and lines joining points represent grouping samples from the same individual and GIT site (buccal mucosa: $\mathrm{n}=18$, dental plaque: $n=23$, dorsum of the tongue: $n=25$, stool: $n=24$ ). Proportion of reads that 
733 were mapped to phage clusters, coloured by viral family, containing only. No convergent

734 solutions were found. Proportion of reads mapped to e) persistent and f) transient phage

735 clusters for individuals in c) and d). "Other" represents non-phage viral families,

736 Alloherpesviridae, Ascoviridae, Baculoviridae, Flaviviridae, Herpesviridae, Iridoviridae,

737 Marseilleviridae, Mimiviridae, Nudiviridae, Phycodnaviridae, Picornaviridae, Pithoviridae,

738 Poxviridae and Retroviridae (see Methods). g) Prevalence of transient and persistent phage

739 clusters in the same individuals ordered by decreasing prevalence of total and persistent

740 phage clusters.

741

742

743

744

745

746

747

748

749

750

751

752

753

754

755

756

757

758 
$\mathbf{a}$

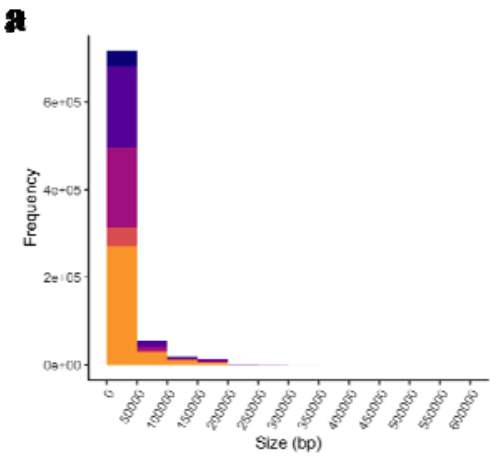

b

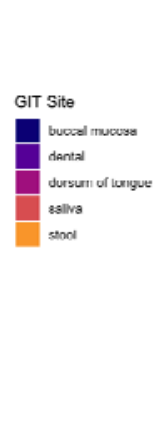

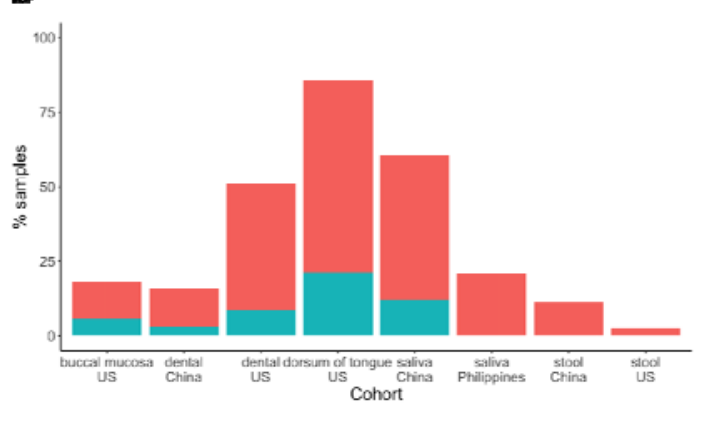

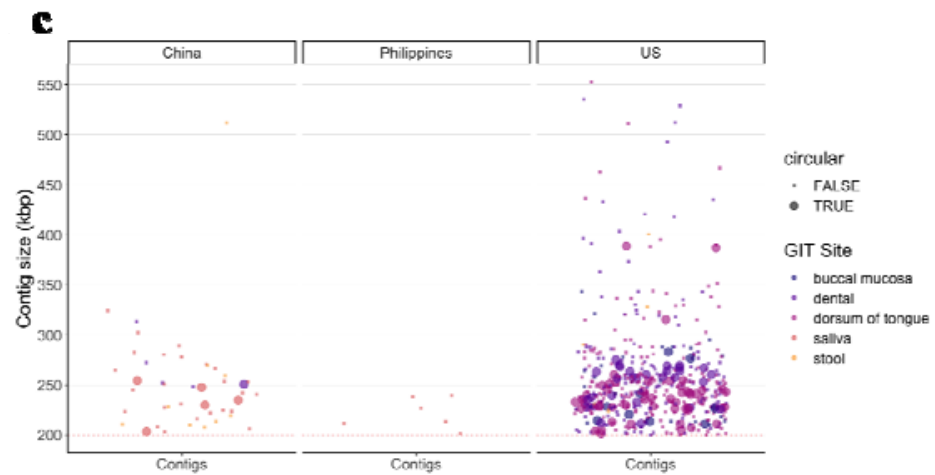

759

d

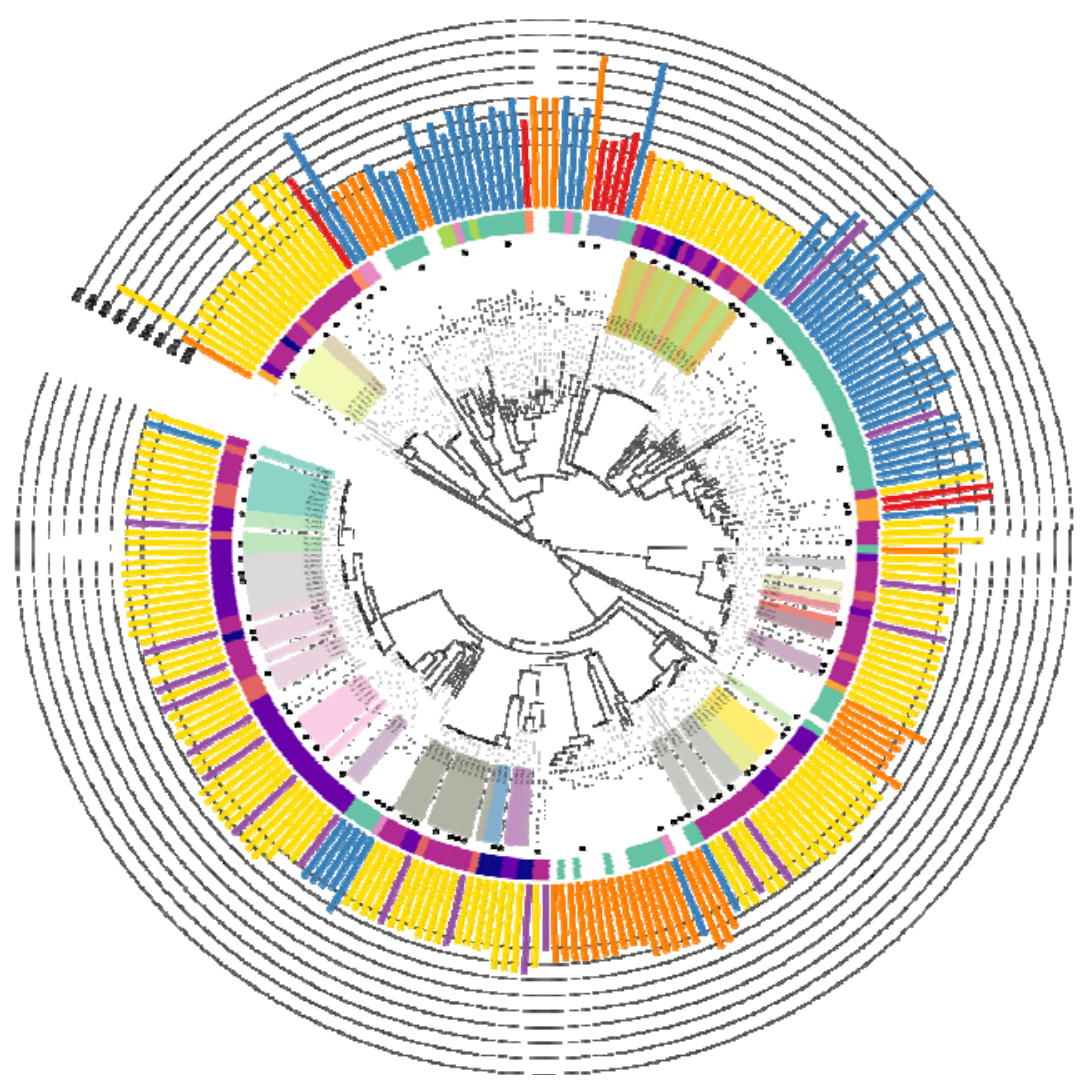

\begin{tabular}{l} 
Environment \\
\hline Adult buccal mucosa \\
Adult dental plaque \\
$\square$ Adult dorsum of tongue \\
Adult fecal samples \\
$\square$ Adult saliva \\
$\square$ Baboon focal samples \\
$\square$ Biotechnology \\
$\square$ Intensive Care Unit \\
$\square$ Outside environment \\
$\square$ Pig fecal samples \\
Study \\
Al-Shayeb et al. \\
$\square$ Devoto et al. \\
$\square$ IMG/NR \\
\hline RefSeq \\
$\square$ This study
\end{tabular}

Figure 4: Prevalence of jumbo phages. a) Histogram of phage genome sizes in bp. b) 
76470 stool), China ( $\mathrm{n}=32$ dental plaque, $\mathrm{n}=33$ saliva and $\mathrm{n}=72$ stool), and the Philippines ( $\mathrm{n}$ $765=24$ saliva). c) Sizes of unique jumbo phage contigs found in cohort. Red dashed line

766 represents $200 \mathrm{~kb}$ cut-off. d) Phylogenetic tree of the major capsid protein (MCP) sequences of jumbo phages from this study $(n=132)$, RefSeq $r 99^{19}(n=37)$, IMG/VR ${ }^{56}(n=18)$, AlShayeb et al. ${ }^{18}(\mathrm{n}=56)$ and Devoto et al. ${ }^{10}(\mathrm{n}=8)$. Labels are coloured by phage cluster. MCPs from circularised jumbo phages that are labelled with black circles at the branch tips. The inner to outer rings show the environment and genome size with study labelled by colour. Tree bootstrapped 1000 times and rooted by midpoint.

$\mathbf{a}$

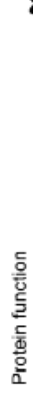

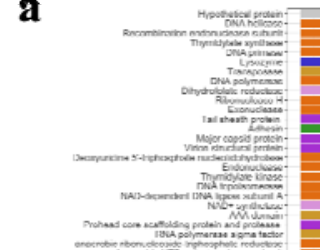

흫
홀
둥
임

778

779 b

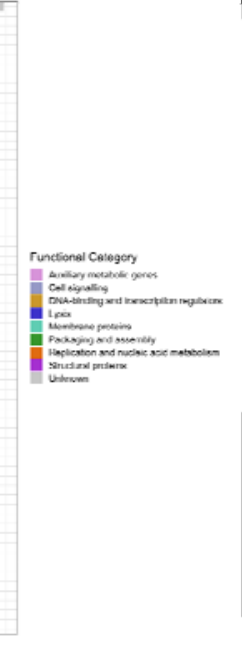

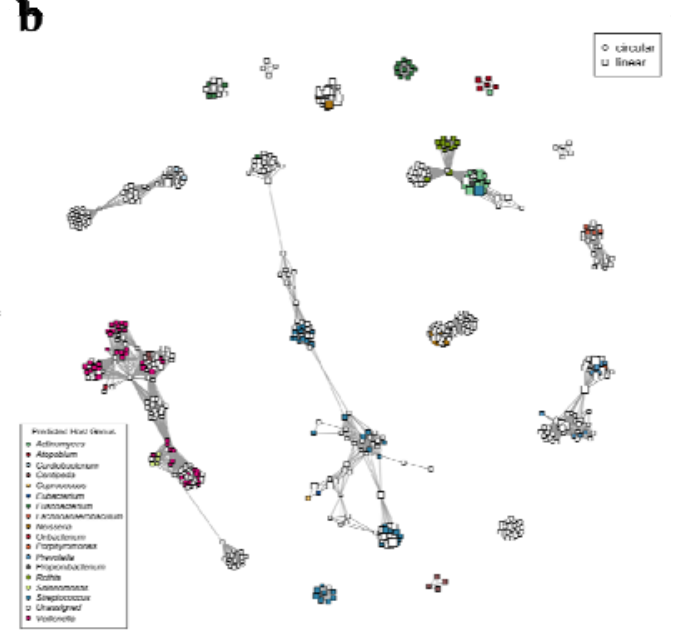

Figure 5: Functions and associations of protein-coding gene in jumbo phages

a) Number of jumbo phages (50 or greater) containing proteins coloured by functional category. Complete list of functional annotations is located in Supplementary Table 3 for each jumbo phage. b) Network of jumbo phages connected by ClusterONE weights of genesharing profiles generated from vContact2. The Centipeda genus represents the C. periodontii species $^{63}$. Full list of jumbo phages can be found in Supplementary Table 2. 